\title{
Analysis of Homogeneous Slurry Pipe Flow
}

R. C. Chen

Department of Mechanical and Marine Engineering National Taiwan Ocean University Keelung 20224, Taiwan,

Republic of China

Follow this and additional works at: https://jmstt.ntou.edu.tw/journal

Part of the Mechanical Engineering Commons

\section{Recommended Citation}

Chen, R. C. (1994) "Analysis of Homogeneous Slurry Pipe Flow," Journal of Marine Science and Technology. Vol. 2:

Iss. 1, Article 5.

DOI: $10.51400 / 2709-6998.2486$

Available at: https://jmstt.ntou.edu.tw/journal/vol2/iss1/5

This Research Article is brought to you for free and open access by Journal of Marine Science and Technology. It has been accepted for inclusion in Journal of Marine Science and Technology by an authorized editor of Journal of Marine Science and Technology. 


\title{
ANALYSIS OF HOMOGENEOUS SLURRY PIPE FLOW
}

\author{
R. C. Chen \\ Department of Mechanical and Marine Engineering \\ National Taiwan Ocean University \\ Keelung 20224, Taiwan, Republic of China
}

Key wards: fluid mechanics, two-phase flow, slurry.

\begin{abstract}
An analysis is performed for the solid-liquid slurry pipe flow in the homogeneous flow regime. The transport and concentration equations are constructed based on two-liquid postulation and modified Rouse-Schmidt diffusion relation. By considering the interphase interaction effects, a general $\mathrm{k}-\in$ model is applied to describe the turbulent kinetic energy production and dissipation. Coupling with the physically-defined parameters, this model is able to obtain the axial velocities of the solid and liquid phases, the solid concentration distribution and the liquid turbulent kinetic energy and dissipation rate. Excellent agreement between the calculated axial velocity profiles and the experimental data obtained by Chen \& Kadambi (1994) is found for the slurry flow with up to $25 \%$ volumetric concentration.
\end{abstract}

\section{INTRODUCTION}

Solid-liquid slurry flows are widely encountered in many industrial, chemical and fossil energy processes. In many of these processes solid concentration up to more than $50 \%$ by volume are encountered. The flow of slurry can be influenced by drag, turbulence, the particle hindered velocity, the virtual mass effect, phase interaction, particle inertia and the crossing-trajectories effect. Basically, four flow regimes can be identified for the solidliquid slurry flow in the horizontal pipe; those are stationary bed flow, saltation flow, heterogeneous flow and homogeneous flow. The experimental investigation of dense slurry flow is far from completion due to the restriction of experimental apparatus. On the other hand, reliable numerical simulation for the slurry flow has not been available yet because of the enormous complexity and the lack of experimental support. The lack of understanding of such slurry flows has resulted in design deficiencies leading to either operational problems like clogging or plugging in many industrial applications. There is a need for more efficient methods of transportation of slurries like coal-water, coal-oil for power stations, metallized liquid fuel for rocketry, ores from the mines etc. A better understanding of the mechanism of such slurry transportation is the first way to reach the goal.

Many numerical simulations have been conducted for solid-liquid slurry flows. Due to the difficulty in dealing with interphase and innerphase (particle-particle) stresses in stationary bed, saltation and heterogeneous flow regimes or dense slurry flows, most of the numerical simulations were restricted to relatively dilute slurry in the homogeneous flow regime. Since turbulence plays dominant role in dilute homogeneous slurry flows, many investigators used this concept to start the development of numerical model (e.g., Elghobashi and Abou-Arab, 1983; Crowder et al, 1984; Michaelides and Farmer, 1984; Roco and Shook, 1985; Persen and Coleman, 1989). The adoption of turbulence model developed for single phase flow in slurry flow, especially in dense slurry, remains unverified. The influence of particle-particle, particle-fluid and particle-wall interactions plays an important role in determining the flow characteristics of dense slurry flows and should be considered more physically in the modelling. At present, due to the complexity, necessary empiricism is needed for the numerical simulation.

From the literature survey, it shows that the applications of the currently existing numerical model is still very limited and it calls for more physical insight of the model. Industrial applications involve many homogeneous slurry pipe flows, the objective of this study is to provide a more physically-clear numerical model for such slurry flows with solid concentration up to $25 \%$ by volume. 


\section{MODELING}

A system of differential equations to obtain axial velocities of the solid and liquid, the solid concentration and the liquid turbulent kinetic energy and dissipation rate for solid-liquid slurry pipe flow in the homogeneous flow regime is developed. In this analysis, each phase is assumed to be a continuum and two-way coupling between the phases is allowed. Note that this assumption is only good for the homogeneous flow regime. Since the particle and fluid phases are assumed to be interacting continua, as a consequence, additional turbulent kinetic energy production and dissipation terms arise and require modeling. A two-equation turbulence model for twophase flows which takes into account the effects of suspended particles on the turbulent structure of the carried liquid is applied. A modified Rouse- Schmidt equation is used to calculate the local concentration distribution. Due to the complexity of this slurry flow, with no exception, some empirical components are involved in this modeling work. The numerical integration is performed by using finite-difference technique. Typical boundary conditions, such as non-slip and impermeable wall and symmetry about pipe center, are applied.

\section{Mathematical equations}

In this analysis of homogeneous slurry flow, both phases are assumed to behave macroscopically as continua and have constant physical properties. The flow is assumed to be incompressible, isothermal, fully developed, steady in the mean but asymmetric due to gravitational effect. Brownian motion and molecular diffusion of the solid phase can be presumed negligible relative to turbulent diffusion for large size particles. While it needs further experimental verification, the turbulence is taken as locally isotropic. With the above assumptions, the continuity and momentum equations can be written for each phase as:

$$
\begin{gathered}
\frac{\partial}{\partial t}\left(C_{\ell} \rho_{\ell}\right)+\frac{\partial}{\partial x_{i}}\left(C_{\ell} \rho_{\ell} V_{\ell i}\right)=0 \\
\frac{\partial}{(\partial t)}\left(C_{k} \rho_{k} V_{k i}\right)+\frac{\partial}{\left(\partial x_{j}\right)}\left(C_{k} \rho_{k} V_{k i} V_{k j}\right) \\
=C_{k} \rho_{k} b_{i}-\frac{\partial}{\left(\partial x_{i}\right)}\left(C_{k} P\right)+\frac{\partial}{\left(\partial x_{j}\right)}\left(C_{k} \tau_{k e j i}\right)+D_{k i}^{s}
\end{gathered}
$$

Designating $k=s, \ell$, for solid and liquid phases, respectively. Stress term $\tau_{\text {keji }}$ caused by viscous, Coulombic, elastic and plastic collision and turbulence can be simplified as

$$
\tau_{k e j i}=\delta_{k \ell} \tau_{k v j i}+\delta_{k s} \tau_{k j j i}+\delta_{k s} \tau_{k c j i}+\tau_{k t j i}
$$

The viscous stress caused by a Newtonian liquid is expressed as

$$
\tau_{\ell v i i}=\mu_{\ell}\left(\frac{\partial V_{\ell i}}{\partial x_{j}}\right)
$$

No viscous stress of the solid phase is assumed. The turbulent stress of both phases may be expressed similar to single-phase flow as

$$
\tau_{k t j i}=-\rho_{k} \overline{\mathbf{v}_{k i}^{\prime} \mathbf{v}_{k j}^{\prime}}=\mu_{k t}\left(\frac{\partial V_{k i}}{\partial x_{j}}\right)
$$

Columbic contact stress (basically, a dynamic stress due to contact friction between particles) is approximated as normal "support load" times a coefficient of dynamic friction (Roco and Shook, 1983). That is

$$
\tau_{k f j i}=\int_{y}^{D} K(y)(S-1) g \rho_{\ell} C_{s} d y=\tau_{k f j j} f_{c}
$$

The interphase stress is found to be a function of slip velocity and can be expressed as (Wen and Yu, 1966)

$$
\begin{aligned}
D_{s i}^{s} & =-D_{l_{i}}^{s} \\
& =\left[\frac{3}{4} C_{d} \frac{C_{s} C_{\ell}\left|V_{\ell i}-V_{s i}\right| \rho_{\ell}}{d_{p} \varphi_{s}} f\left(C_{\ell}\right)\right]\left(V_{\ell i}-V_{s i}\right)
\end{aligned}
$$

Note that $C_{d}$, the drag coefficient of a free-fall particle, is related to particle Reynolds number $\mathrm{Re}_{s}\left(=\mathrm{V}_{\mathrm{o}} \mathrm{d}_{\mathrm{p}} / \nu_{\ell}\right)$ as

$$
\begin{array}{cc}
\mathrm{C}_{d}=18.5 \operatorname{Re}_{s}^{-0.6} & 0.1<\operatorname{Re}_{\mathrm{s}}<500 \\
\mathrm{C}_{d}=0.44 & 500<\operatorname{Re}_{s}<2 \times 10^{5}
\end{array}
$$

The empirical function $f\left(C_{\ell}\right)$ which accounts for the collective effect of the presence of particles in the fluid and acts as a correction to the usual Stokes law for free fall of a single particle can be expressed as (Wallis, 1969)

$$
f\left(C_{\ell}\right)=C_{l}^{-2.65}
$$

Starting from the concept of the kinetic energy of solid, the dispersive stress due to the elastic and plastic collisions of solids may be correlated to the turbulent shear stress. By considering the significant dispersive effect for $i=j$, Roco and Balakrishnam (1985) proposed the expression for the predominant parts of spatial gradient of the collision stress as 


$$
\begin{aligned}
& \frac{\partial}{\partial x_{j}}\left(C_{s} \tau_{s c j i}\right) \\
& \simeq \frac{-\left(1-\delta_{i j}\right)}{\tan \theta} \frac{\partial}{\partial x_{i}}\left(\rho_{s} C_{s} \overline{v_{s i}^{\prime} v_{s j}^{\prime}}\right)
\end{aligned}
$$

where $\tan \theta$ is the coefficient of dynamic friction between solid particles and has the value between 0.3 and 0.75 (Bagnold, 1954). In determining the local concentration distribution, the interactions between solid particles are assumed to be negligible compared with the turbulence effect. Therefore, a turbulent form of Schmidt-Rouse diffusion expression is used in this analysis as

$$
\begin{array}{r}
\frac{\partial C_{s}}{\partial t}+\frac{\partial}{\partial x_{j}}\left[C_{s} V_{s j}+\overline{C_{s}^{\prime} v_{s j}^{\prime}}\right] \\
=\varepsilon_{s} \frac{\partial^{2} C_{s}}{\partial x_{j}^{2}}+\frac{\partial \varepsilon_{s}}{\partial x_{j}} \frac{\partial C_{s}}{\partial x_{j}}
\end{array}
$$

From the experimental results, the eddy diffusivity of particles can be expressed as (Roco and Shook, 1987)

$$
\varepsilon_{\mathrm{s}}=0.146 V_{\tau} \frac{\rho_{\mathrm{l}}}{\rho_{s}} \mathrm{y}\left(2-\frac{2 \mathrm{y}}{D}\right)\left[\left(1-\frac{2 y}{D}\right)^{2}+0.54\right]
$$

Hindered settling velocity $\left(\mathrm{V}_{\mathrm{m}}\right)$ which only exists in the direction of gravity is assumed to be independent of position and can be written as (Richardson and Zaki, 1954)

$$
V_{s j}=V_{m}=V_{o}\left(1-C_{s}\right)^{m}
$$

with $\mathrm{m}=4.45 \operatorname{Re}_{\mathrm{m}}{ }^{-0.1}$ when $\left.1<\operatorname{Re}_{\mathrm{m}}\left(=\mathrm{V}_{\mathrm{m}} \mathrm{d}_{\mathrm{p}} / \nu_{\mathrm{l}}\right)\right)$ $<500$ and $m=2.39$ when $\operatorname{Re}_{\mathrm{m}}>500$. Analogous to the velocity fluctuation, the turbulent component of mass flux is simplified by the expression of the product of particle turbulent viscosity and concentration gradient. Simply,

$$
\overline{\mathrm{v}_{s j} c_{s}^{\prime}}=-v_{s t} \frac{\partial C_{s}}{\partial x_{j}}
$$

For turbulent structure, the two-equation $(\mathrm{k}-\epsilon$ ) model described by Elghobashi and Abou-Arab (1983) is used as the foundation for this study. This model is written as

(a) k equation

$$
\begin{aligned}
& \frac{\partial}{\partial t}\left(C_{\ell} \rho_{\ell} k\right)+\frac{\partial}{\partial x_{j}}\left(C_{\ell} \rho_{\ell} k V_{\ell j}\right) \\
& =\frac{\partial}{\partial x_{j}}\left(C_{\ell} \rho_{\ell}\left(v_{\ell}+\frac{v_{\ell t}}{\sigma_{k}}\right) \frac{\partial k}{\partial x_{j}}\right)+C_{\ell} \rho_{\ell} v_{\ell t}\left(\frac{\partial V_{l t}}{\partial x_{j}}\right)^{2}
\end{aligned}
$$

$-\rho_{\ell} C_{l} \in$

+ extra production + extra dissipation

(b) $\in$ equation

$$
\begin{aligned}
& \frac{\partial}{\partial t}\left(C_{\ell} \rho_{\ell} \in\right)+\frac{\partial}{\partial x_{j}}\left(C_{\ell} \rho_{\ell} k V_{\ell j}\right) \\
& =\frac{\partial}{\partial x_{j}}\left(C_{\ell} \rho_{\ell}\left(v_{\ell}+\frac{v_{\ell}}{\sigma_{\epsilon}}\right) \frac{\partial \epsilon}{\partial x_{j}}\right) \\
& +C_{\epsilon 1}\left(\frac{\epsilon}{k}\right) C_{\ell} \rho_{\ell} v_{\ell}\left(\frac{\partial V_{j}}{\partial x_{j}}\right)^{2}-\rho_{\ell} C_{\ell} C_{\in 2} \frac{\epsilon^{2}}{k} \\
& + \text { extra production + extra dissipation }
\end{aligned}
$$

The extra production and dissipation terms in the above equations are due to the change of turbulence structure of liquid phase caused by the interaction between two phases. The turbulent correlations become much more complex because of the presence of particles. The change of turbulence structure can be observed mathematically from the terms of turbulent correlation equations which involve correlations of solid concentration with velocity fluctuations, pressure, strain rate and so on. The group of solid concentration correlations composes the extra production and dissipation terms in the final $\mathrm{k}-\epsilon$ equations (Elghobashi and Abou-Arab, 1983; Crowder et al., 1984). There are more that 60 terms involved in the extra dissipation and production terms to account for the change of turbulence structure of liquid phase caused by the interaction between solid particles and liquid. A simplification of these equations is necessary. In the final form, the turbulent viscosity of solid phase is approximated as suggested by Peskin (1962) by

$$
\frac{v_{s t}}{v_{l t}}=1-\frac{\Gamma_{\ell}^{2}}{15} \frac{\epsilon}{v_{l}} \frac{3 k^{2}}{k+2}
$$

Also, the dominant terms for extra production plus extra dissipation in $k$ and $\in$ equations are respectively found to be

$$
-\frac{\rho_{s} C_{\ell}}{\tau_{m}} C_{s} \mathrm{k}\left(\frac{2}{3}-\frac{2 \Gamma_{\ell}}{\tau_{m}+\Gamma_{l}}\right)
$$

and

$$
\begin{aligned}
& -\frac{\rho_{s} C_{\ell}}{\tau_{m}} C_{s}\left[\epsilon-v_{\ell}\left[\frac{\epsilon}{v_{\ell}} \frac{\Gamma_{\ell}}{\tau_{m}+\Gamma_{\ell}}\right.\right. \\
& \left.\left.+\frac{\tau_{m}}{\left(\tau_{m}+\Gamma_{\ell}\right)^{2}}\left(\frac{\partial \mathrm{k}}{\partial x_{j}} \frac{\partial \Gamma_{\ell}}{\partial x_{j}}\right)\right]\right]
\end{aligned}
$$




\section{Numencal algorithm}

A finite difference numerical technique in the Cartesian coordinate is used for solving the non-linear forms of main differential equations. There is no advantage of using the cylindrical coordinate because of the natural asymmetric flow field in the slurry flows (although we call it "homogeneous" flow). A Eulerian mesh of finite-difference cells is used for representation of the governing equations. A typical central-difference method is utilized for the computational domain except on the wall boundary where either the forward or backward difference formula is applied. In one-dimensional pipe flows the pressure gradient usually is prescribed. The empirical pressure loss relations suggested by Turian and Yuan (1977) by correlating over 4000 slurry flow test data available in the literature are used in the numerical simulation for a wide range of application. Non-slip, impermeable and wallshear conditions at wall for both phases and symmetric conditions at pipe centerline are implemented as boundary conditions. Due to the unavailability of experiment-based turbulence constants for slurry flows, the constants applied for single phase flow (Patel et al., 1985) are used in this investigation. Further experimental studies are recommended for verifying this adoption. A summary of the constants used in this study is given in Table 1.

The main calculation is performed on IBM-4361 computer. Guesses based on the single- phase solution are input as the initial conditions. The criterion of a maximum $0.1 \%$ difference between two consecutive iterated quantities is chosen for closure.

\section{RESULTS}

The numerical procedure is first examined with reference to the single phase flows by setting the solid concentration as zero. These computations serve as the calibration of the numerical method. Excellent agreement between the numerical data of present study and the experimental data obtained by Chen and kadambi (1994) is observed. Excluding the region $5<\mathrm{Y}^{*}<70$ where there is an overlap of turbulent core and wall shear flow regions and results in large scatter of experimental data, good agreement for turbulent kinetic energy and dissipation rate between computed results and other researchers' experi-

Table 1 . Constants used in numerieal model

\begin{tabular}{ll}
$\varphi_{\mathrm{s}}$ & 1 \\
$\tan \theta$ & 0.5 \\
$\mathrm{c}_{\mathrm{u}}$ & 0.09 \\
$\sigma_{\mathrm{k}}$ & 1 \\
$\sigma_{\in}$ & 1.3 \\
$\mathrm{C}_{\in 1}$ & 1.44 \\
$\mathrm{C}_{\in 2}$ & 1.92 \\
$\mathrm{C}_{\mathrm{t}}$ & 0.41 \\
\hline
\end{tabular}

mental and numerical efforts (Patel et al., 1985) is also found.

Figure 1 shows the calculated flow properties of different concentration slurry flow along the horizontal diameter as a function of wall distance. Dimensionless forms of axial velocities $\left(\mathrm{U}^{+}=\mathbf{u} / \mathbf{u}_{\tau}\right)$, dissipation rate of turbulence $\left(\epsilon^{*}=v_{\ell} \in h_{\tau}\right)$, turbulent kinetic energy $\left(k^{*}=k / u_{\tau}{ }^{2}\right)$ and wall distance $\left(\mathrm{Y}^{+}=\mathrm{yu}_{\tau} / v\right)$ are used in Fig. 1. Compared with the single phase flow, the dissipation rate of turbulence for slurry flow increases substantially near the
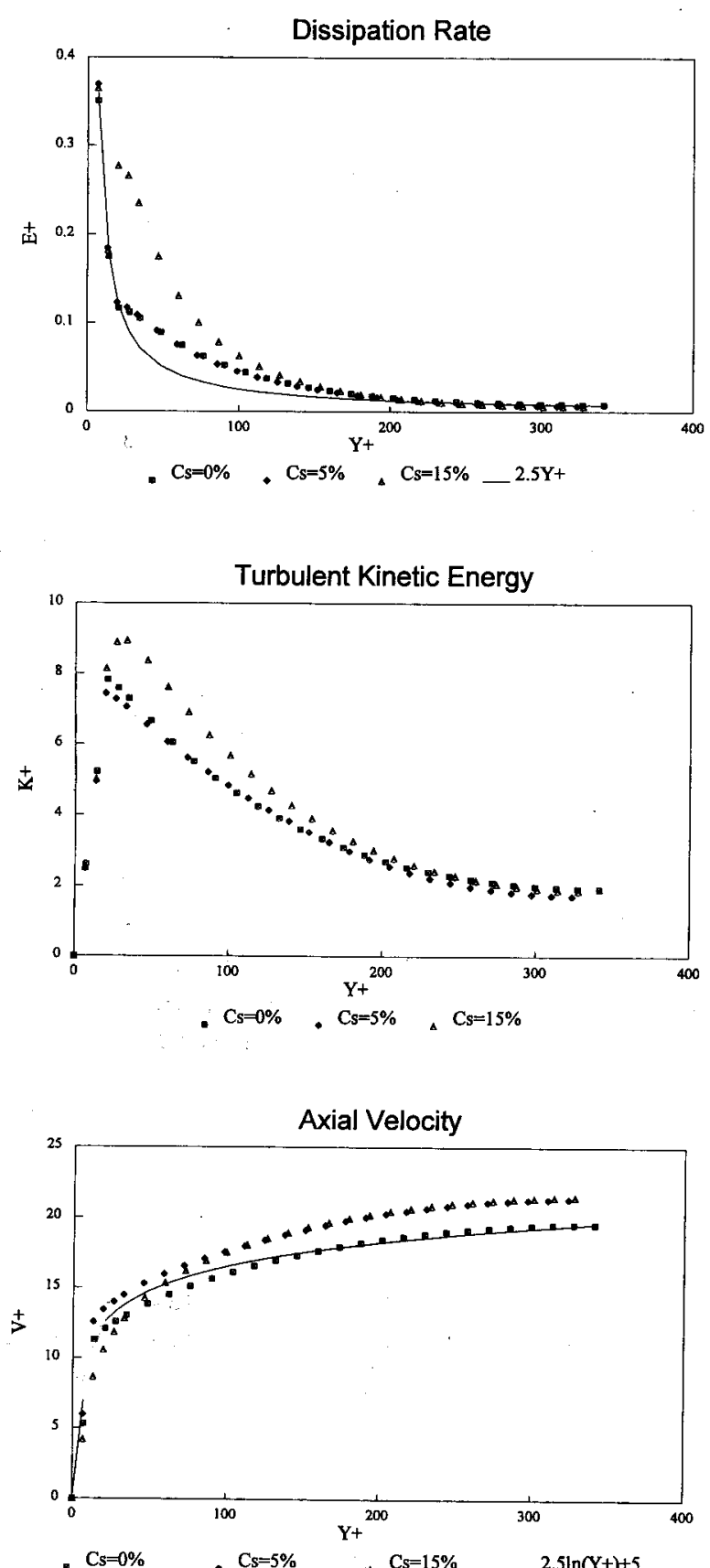

Fig. 1. Dimensionless flow properties of different concentration slurry flows along the diameter at a Reynolds number of 10150. 
wall region but decreases slightly in the core region. Despite the small change for $5 \%$ slurry flow, the turbulent kinetic energy of both $5 \%$ and $15 \%$ slurry flows is also observed to increase in the region near the wall and decrease in the core region. The increase of the turbulence level is smaller than that of the energy dissipation rate which are both caused by the interaction of the solid phase. The uneven increase of dissipation rate and kinetic energy results in the change of turbulence structure, especially in the region near the wall. The phenomena of enlarged wall shear region is obvious as shown in Fig. 1 of which velocity profile is "distorted" by "pulling down" of the velocity near wall and "pushing up" of the velocity close to the turbulent core.

The calculated distribution of concentration is uniform along the horizontal diameter. However, even though the flow regime is homogeneous, there is a small concentration gradient along the vertical diameter as shown in Figure 2. This concentration gradient becomes smaller with the increase in Reynolds number. The concentration gradient along the vertical diameter is introduced by the consideration of gravitational effect, shown in hindered settling velocity term, in the concentration equation. On
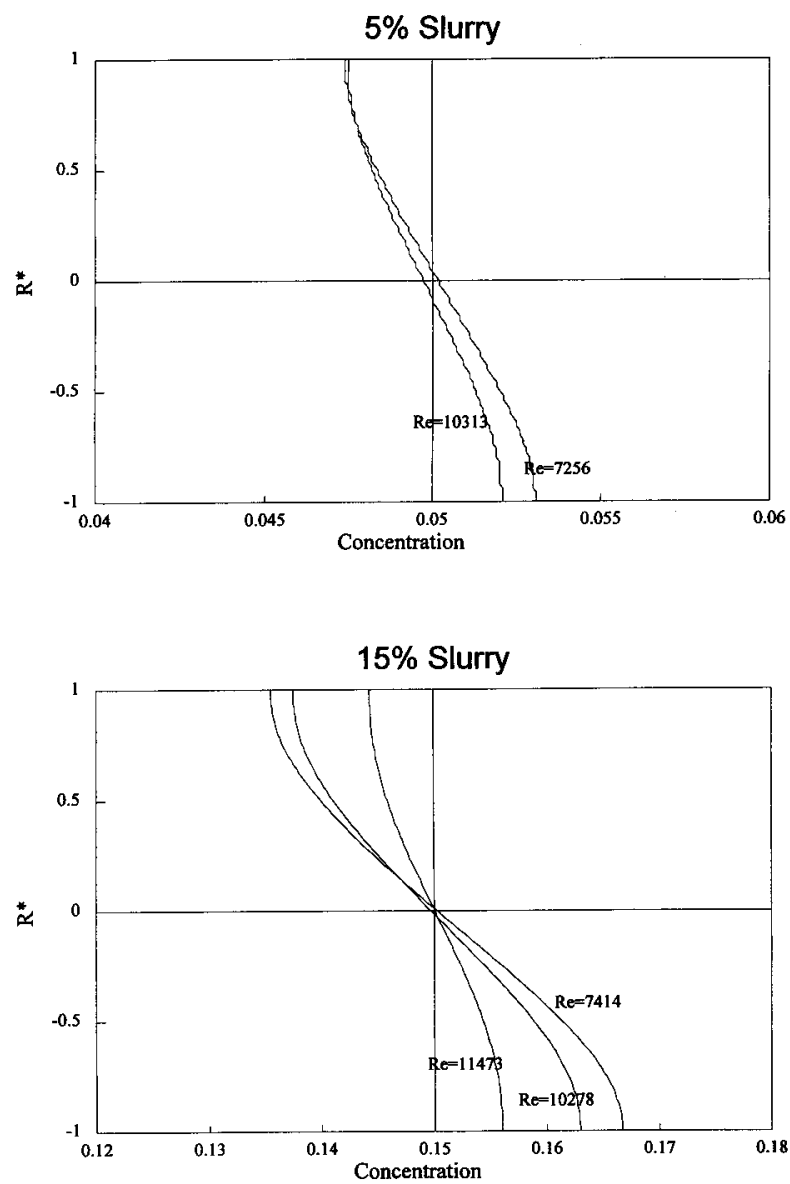

Fig. 2. Concentration profiles along the horizontal and vertical diameters for different concentration slurry flows at various Reynolds numbers. the other hand, as the Reynolds number increases, turbulent effect becomes dominant over gravity and results in the decrease in the concentration gradient along the vertical diameter.

The following comparison of the computed and experimental results is performed with the LDV data obtained by Chen and Kadambi (1994). Comparison of axial velocity profiles is presented in Figure 3 for $5 \%$ and $15 \%$ slurry flows. Excellent agreement can be observed in this figure. The calculated velocity profiles also agree with the experimental data along several different vertical and horizontal lines as shown in Figure 4 for instance. It should be noted that the numerical results give very small slip velocity $(<0.5 \%)$ between solid and liquid phases for all different concentration slurry in the homogeneous flow regime. Also the calculated velocity distributions are similar along the horizontal and vertical diameters. Therefore, the results shown here represent velocities of both solid and liquid phases along the horizontal and vertical diameters.

Figure 5 shows the calculated flow properties and the comparison of the numerical and experimental data for $25 \%$ slurry flow. In this figure, the distortion of the velocity profile due to the existence of particles becomes more remarkable. The extra shear stresses introduced by interactions of particle-particle, particle-liquid and particlewall consequently enlarge the wall shear flow region and distort velocity profiles. Obviously, this extra stresses increase with solid concentration. Compared with $15 \%$ slurry flow case, both the turbulent kinetic energy and the dissipation rate of turbulence increase substantially. Near the wall region, the increase in the dissipation rate, however, is much larger than in the kinetic energy. This phenomena may account for the remarkable enlargement of wall shear region. Relatively large concentration gradient along the vertical diameter can be also seen in Fig. 5. Figure 5 also reveals that, instead of the mean value $(25 \%)$ as in the cases of 5 and $15 \%$ slurry flows, the $25 \%$ slurry maintains at a slightly higher concentration $(\approx 25.5 \%)$ with a small gradient along the horizontal diameter. It implies that the gravitational effect becomes relatively significant for $25 \%$ slurry flow under the similar Reynolds number range. Comparison of numerical velocity profiles and experimental data is also satisfactory for the $25 \%$ slurry flow case. Unfortunately, for slurry flow over $25 \%$ solid concentration, poor agreement resulting from the under-estimation in turbulent core and over-estimation in wall region occurs between the numerical and experimental data. It is not surprising because that most of the interphase stress and mass diffusion expressions were developed under the assumption of low concentration condition, the application of these semi-empirical-expressions are then limited to "dilute" solution. Additionally, the assumptions of zero solid molecular viscosity and isotropic turbulence structure are not proper for such dense slurry flows. Not until such key questions can be 


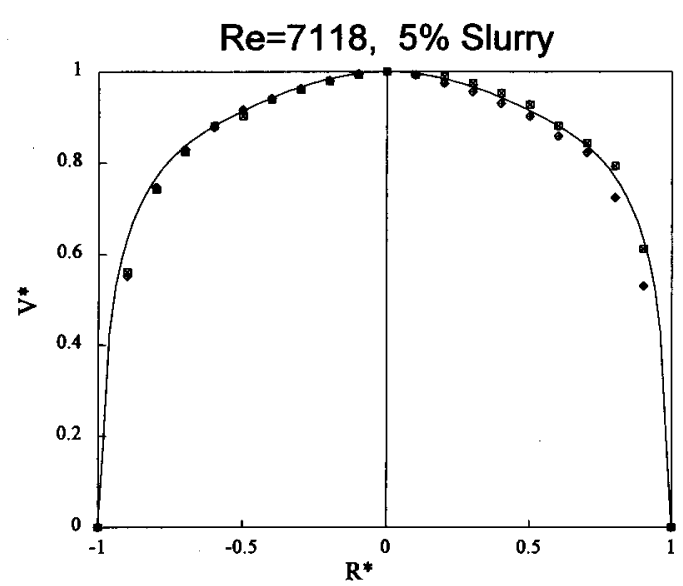

- Exp. data (h) . Exp. data (v) _ Num. data

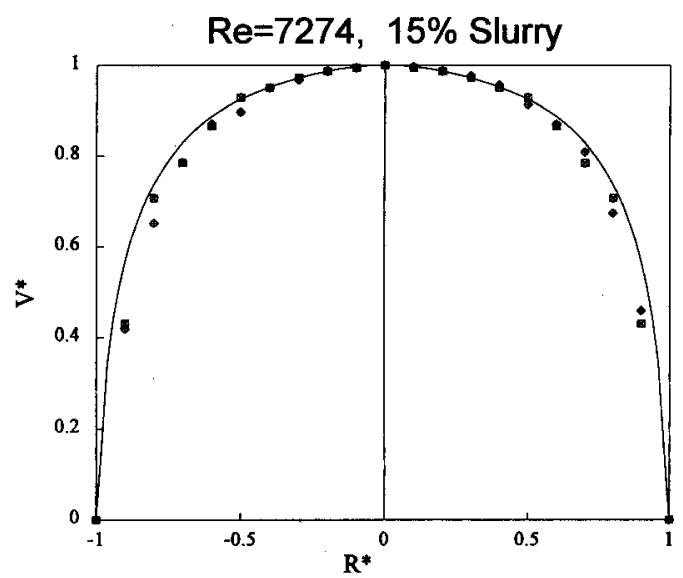

- Exp. data (h) _Exp. data (v) _Num. data
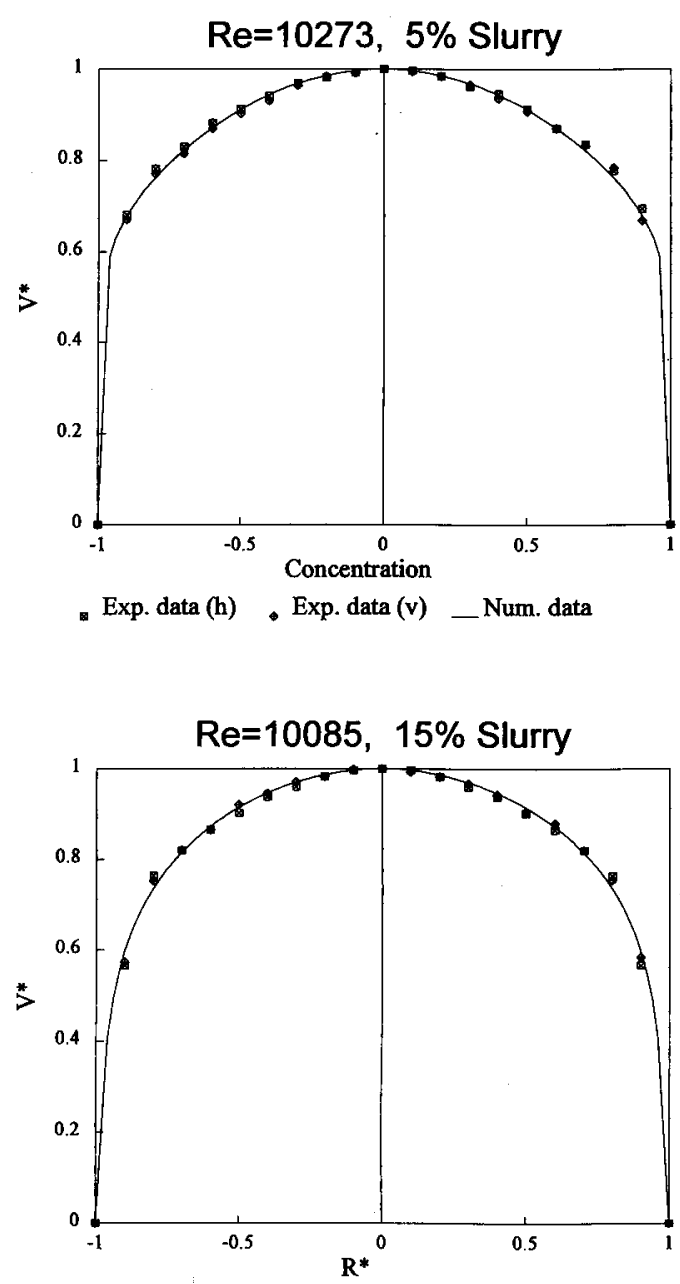

. Exp. data (h) _. Exp. data (v) _._Num. data

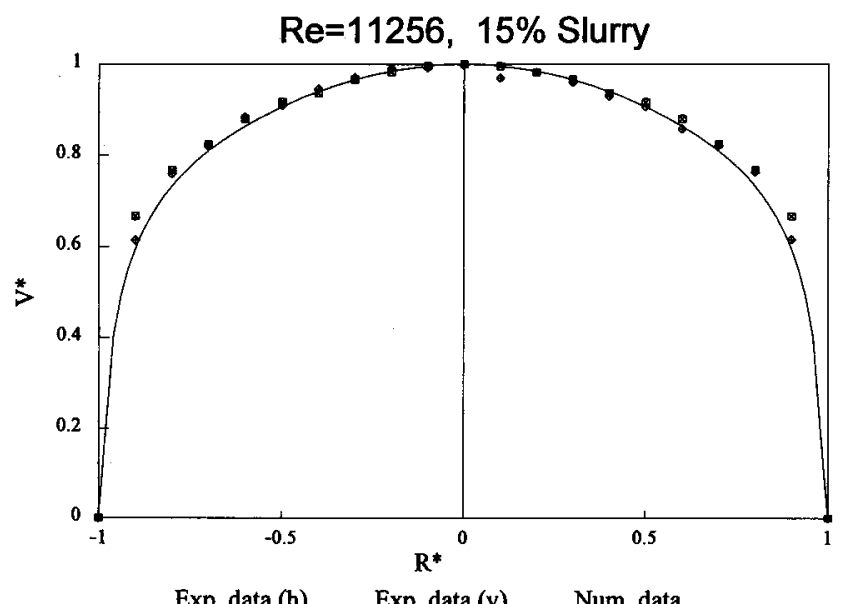

Fig. 3. Comparison of numerical and experimental axial velocity datea for different concentration slurry flows at various Reynolds numbers. 

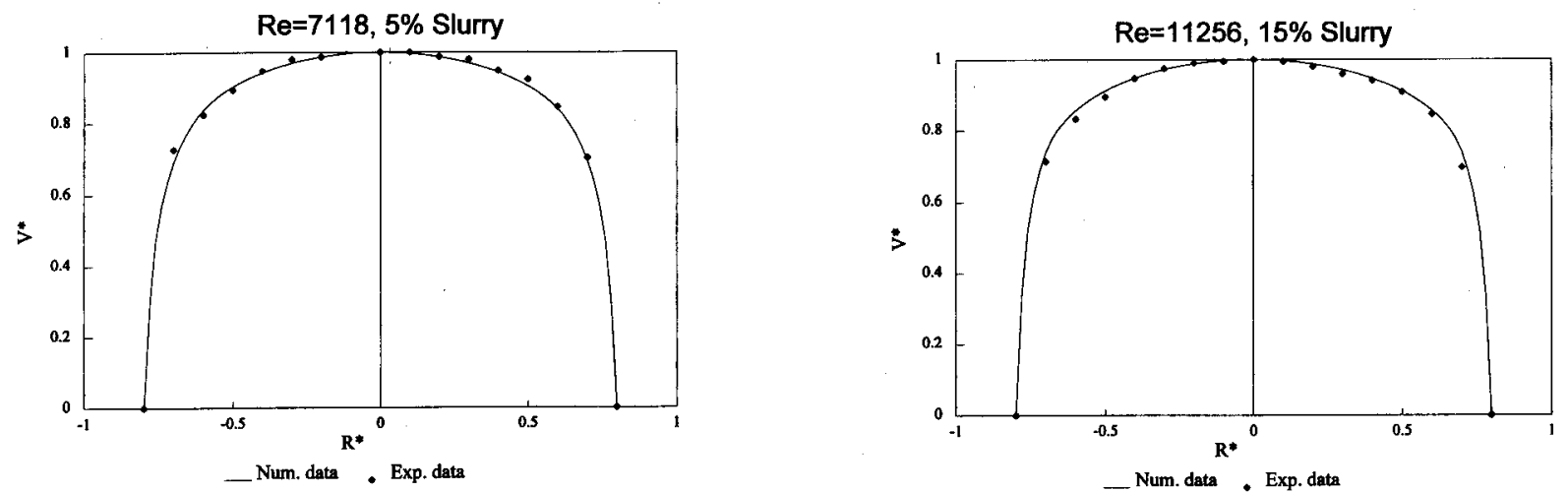

Fig. 4. Comparison of numerical and experimental axial velocity data along vertical line $7.5 \mathrm{~mm}$ left to the vertical diameter for different concentration slurry flows at various Reynolds numbers.
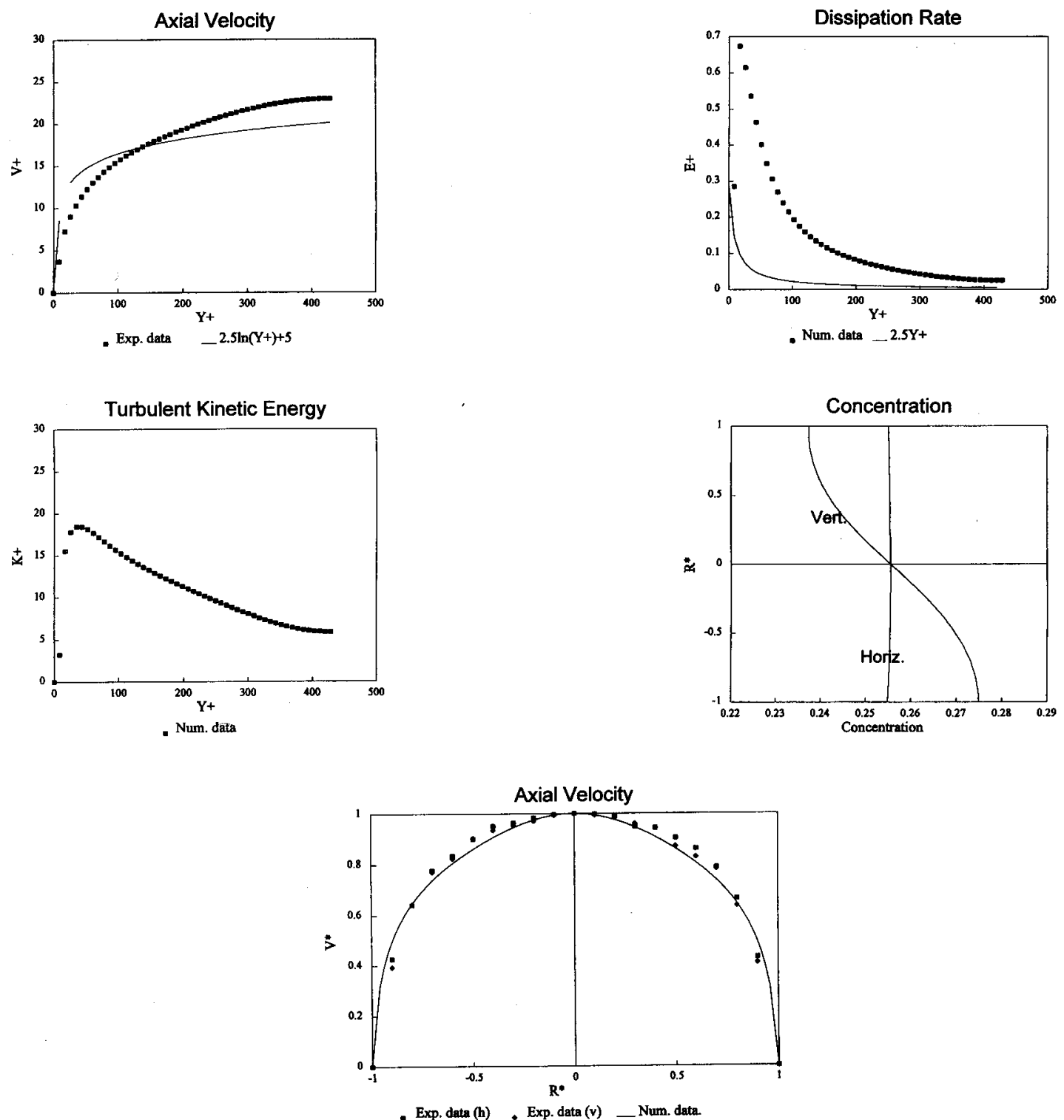

Fig. 5. Dimensionless flow properties of $25 \%$ slurry flow along the diameter at a Reynolds number of 13359. 
answered will the understanding and modeling of the dense slurry flows be improved.

\section{CONCLUDING REMARKS}

An analysis based on transport equations including mass, momentum and turbulence energy and empirical coefficients for phase interactions is performed for homogeneous slurry pipe flow. The result shows that extra energy dissipation and production caused by the particle interphase and innerphase interactions result in the enlargement of wall shear region and the reduction of turbulence core and therefore accounts for the distortion of axial velocity profiles. The comparison with the experimental data shows excellent agreements for solid concentration up to $25 \%$ by volume. Disagreement between numerical results and experimental data in the wall region, however, is observed for slurry flow with volumetric concentration over $25 \%$.

\section{REFERENCES}

1. Bagnold, R. A., 1954, "Experiments in a Gravity Free Dispersion of Large Solid Spheres in a Newtonian Fluid Under Shear", Proc. Roy. Soc., A225, pp 49-63.

2. Chen, R. C. and J. R. Kadambi, 1994, "Discrimination Between Solid and Liquid Velocities in Slurry Flow Using LDV", Measurement Sci. \& Tech., in review.

3. Crowder, R. S., Daily, J.W. and Humphrey, J. A. C., 1984, "Numerical Calculation of Particle Dispersion in a Turbulent Mixing Layer Flow", Energy Sources Technology Conference, ASME, pp 16-26.

4. Elghobashi, S. E. and Abou-Arabj T.W., 1983, "A Two-Equation Turbulence Model for Two-Phase Flows", Phys. Fluids, v. 26, no. 4, pp 931-938.

5. Michaelides,E. E., Farmer, L. K., 1984, "A Model for Slurry Flows Basedon the Equations of Turbulence", Energy Sources Technology Conference, ASME, pp 33-40.

6. Patel, V. C., Rodi, W. and Scheuerer, G., 1985, "Turbulence Models for Near-Wall and Low Reynolds Number Flows: A Review, AIAA J., v. 23, no. 9, pp 1308-1319.

7. Person, L. N. and Coleman, N.L., 1989, "The Influence of Suspended Particles on Turbulent Shear Flow", 3rd Joint ASCE/ASME Mechanics Conference, pp 81-86.

8. Peskin, R.L., 1962, "The Diffusivity of Small Particles in Turbulent Fluids", National Meeting AIChE, Baltimore, MA.

9. Roco, M. C. and Shook, C. A., 1983, "Modeling of Slurry Flow: The Effect of Particle Size", Canadian J. Chem. Eng., v. 61, pp 494-503.

10. Roco, M. C. and Shook, C. A., 1985, "Turbulent Flow of Incompressible Mixtures", J. Fluid Eng., v. 107, pp 224-231.
11. Roco, M. C. and Balakrishnam, N., 1985, "MultiDimensional Flow Analysis of Solid-Liquid Mixtures", J. Rheology, v. 29, no. 4, pp 431456.

12. Roco, M. C. and Shook, C. A., 1987, "New Approach to Predict Concentration Distribution in Fine Particle Slurry Flows", Physicochemical Hydrodynamics, v. 8, no. 1, pp43-60.

13. Richardson, J. F. and Zaki, W. N., 1954, "Sedimentation and Fluidization", Trans. Ins. Chem. Engrs., 32, pp 35-53.

14. Turian, R. M. and Yuan, T., 1977, "Flow of Slurries in Pipelines", AIChE J., v. 23, no. 3, pp 232-243.

15. Wallis, G. B., 1969, "One Dimensional Two-Phase Flow", McGraw-Hill, New York.

16. Wen, C. Y. and Yu, Y. H., 1966, "Mechanics of Fluidization", Chem Eng. Prog. Symp. Ser., 62, pp 100.

17. Wilson, W., 1942, "Mechanics of Flow with NonColloidal Solids", Trans. ASCE, 107, pp 108-113.

\section{NOMENCLATURE}

$\begin{array}{ll}\mathrm{b} & \text { Body force } \\ \mathrm{C}, \mathrm{c}^{\prime} & \text { Mean \& fluctuation component of concen- } \\ & \text { tration } \\ \mathrm{C}_{\mathrm{d}} & \text { Drag coefficient } \\ \mathrm{C}_{\varepsilon 1}, \mathrm{C}_{\varepsilon 2}, \mathrm{C}_{\mathrm{t}}, \mathrm{c}_{\mathrm{u}} & \text { Constant in } \mathrm{k}-\epsilon \text { model } \\ \mathrm{D} & \text { Diameter of pipe } \\ \mathrm{D}^{s} & \text { Interphase stress } \\ \mathrm{d} & \text { Diameter of particle } \\ \delta & \text { Delta function } \\ \epsilon & \text { Dissipation rate of turbulence } \\ \varepsilon & \text { Mass diffusivity } \\ \mathrm{f}_{\mathrm{c}} & \text { Coefficient of dynamic friction } \\ \mathrm{g} & \text { Gravity acceleration } \\ \mathrm{K} & \text { Coefficient of support load } \\ \mathrm{k} & \text { Turbulent Kinetic energy } \\ k & \text { Ration of particle response time to fluid } \\ \mu, v & \text { Lagrangian time scale }\left(=2 \tau_{\mathrm{m}} / \Gamma_{\ell}\right) \\ \varphi & \text { Molecular \& kinematic viscosity } \\ \mathrm{P} & \text { Shape factor } \\ \mathrm{Re} & \text { Pressure } \\ \rho & \text { Reynolds number } \\ \mathrm{S} & \text { Density } \\ \sigma_{\mathrm{k}}, \sigma_{\epsilon} & \text { Density ratio }\left(=\rho_{\mathrm{s}} / \rho_{\ell}\right) \\ \tan \theta & \text { Constant in k- } \epsilon \text { model } \\ \Gamma & \text { Coefficient of dynamic friction between } \\ \tau & \text { solid particles } \\ \tau_{\mathrm{m}} & \text { Lagrangian time scale }\left(=\mathrm{C}_{\mathrm{t}} \mathrm{k} / \epsilon\right) \\ \mathrm{u}, \mathrm{V}, \mathrm{v} & \text { Stress } \\ \mathrm{V}_{\mathrm{m}}, \mathrm{V}_{\mathrm{o}} & \text { Particle response time }\left(=\rho_{\mathrm{s}} \mathrm{d}_{\mathrm{p}}{ }_{\mathrm{p}} / 18 / \mu_{\ell}\right) \\ & \text { Mean \& fluctuation component of velocity } \\ & \text { Hindered velocity }\left(\mathrm{V}_{\mathrm{o}}=\rho_{\ell}(\mathrm{S}-1) \mathrm{d}_{\mathrm{p}}{ }^{2} \mathrm{~g} / 18 / \mu_{\ell}\right) \\ & \end{array}$


c

f

i, j

k

l

$\mathrm{p}$
Elastic and plastic component

Coulombic component

Direction

Phase

Liquid phase

Particle s

t

$\mathrm{v}$

$\mathrm{x}, \mathrm{y}, \mathrm{z}$

$\tau$
Solid phase

Turbulent component

Viscous component

Direction

Shear component 